\title{
Avaliação de Desempenho do IEEE 802.11 para Coleta de Grande Volume de Dados em Florestas com Auxílio de Drones
}

\author{
Caroline Maul de A. Lima ${ }^{1}$, Eduardo A. da Silva ${ }^{1}$, Pedro B. Velloso ${ }^{1}$ \\ ${ }^{1}$ Grupo de Teleinformática e Automação - GTA/COPPE \\ Universidade Federal do Rio de Janeiro - UFRJ
}

\begin{abstract}
Most IoT solutions, in order to reduce power consumption, assume severe constraints on the transmission rate, which limit their use to applications with small amounts of collected data per device. Thus, these solutions are not suitable for monitoring wild animals, which requires transmitting large amounts of photos captured by cameras, installed in the middle of the forest. In addition, the forest environment also impairs the transmission capacity. Thus, this work proposes collecting data from devices in the forest with the aid of drones. Hence, this paper aims at evaluating the performance of a wireless network between the device attached to the drone and the cameras. To achieve our goal, we evaluate traditional network metrics, such as maximum range, transmission rate, and packet loss. To compare and better characterize the transmission in the forest, we also consider different scenarios. The most important result is the feasibility of using low-complexity devices for forest applications.
\end{abstract}

Resumo. A maioria das soluções para IoT, com intuito de reduzir o consumo de energia, assume severas restrições à taxa de comunicação limitando-as para o uso de aplicações onde os sensores captam pequenas quantidades de dados. Por isso, estas soluções não são adequadas para o monitoramento de animais silvestres, que exige a transmissão de grandes quantidades de fotos capturadas por câmeras trap instaladas no meio das florestas. Além disso o ambiente florestal também prejudica a capacidade de transmissão. Portanto, este trabalho propõe a coleta de dados de dispositivos na floresta com o auxílio de drones. Assim, este artigo visa avaliar o desempenho de uma rede sem fio para a comunicação entre o drone e os dispositivos de captura de imagem. Para tal, são avaliadas as principais métricas de rede, tais como alcance máximo, taxa de transmissão e perda de pacotes. A título de comparação e para melhor caracterizar a transmissão na floresta, são realizados testes em outros cenários. $O$ resultado mais importante é a viabilidade do uso desses tipos de dispositivos de baixa capacidade computacional para aplicações florestais.

\section{Introdução}

Tradicionalmente, o monitoramento de animais silvestres é realizado através de câmeras que são instaladas no interior das florestas, em lugares previamente definidos, a fim de registrar a presença deste animais através de fotos ou vídeos [Steenweg et al. 2017]. Para tanto, estas câmeras, comumente denominadas $c a-$ mera trap (câmera armadilha), são dotadas de um sensor de presença que dispara a câmera assim que detecta a presença de algo. Apesar de amplamente utilizado, este procedimento 
de monitoramento implica um elevado custo operacional de instalação das câmeras e sobretudo de coleta dos dados. Em geral, as áreas de monitoramento se localizam em unidades de conservação, em regiões extensas e de difícil acesso. Usualmente, a coleta de dados é realizada de forma puramente manual que exige a presença de pessoas para recuperar cada uma das câmeras e retirar os dados. Além de representar um custo bastante elevado, este método não é nada eficiente, visto que não leva em conta, por exemplo, se a câmera que será visitada tirou alguma foto, se ela apresentou algum defeito, ou mesmo se ainda estava com bateria. Por fim, dependendo do tamanho do parque e da área monitorada, este procedimento pode levar muitos dias. Portanto, este trabalho propõe um sistema automatizado de coleta de dados para o monitoramento de animais silvestres em unidades de conservação.

A ideia consiste em aplicar o conceito de IoT, ou seja, dotar as câmeras com a capacidade de comunicação com dispositivos de baixo custo, de modo a permitir o envio das fotos para um drone que sobrevoará a floresta coletando as fotos de cada uma das câmeras. No entanto, tradicionalmente, as aplicações de IoT assumem que os dispositivos de captura de dados coletam pequenas quantidades de informação, como por exemplo, temperatura, pressão, umidade, posição e assim por diante. Assim, estes dispositivos não precisam ter uma alta capacidade de transmissão. Esta premissa vai ao encontro da limitação de consumo de energia destes dispositivos. Para comprovar este fato, muitas das propostas mais recentes para IoT, como Lora ${ }^{1}$ e $\operatorname{SigFox}^{2}$, preveem a duração da bateria da ordem de anos, com uma taxa de transmissão bastante reduzida. Claramente, este tipo de tecnologia de comunicação não é adequada para aplicações de grande quantidade de dados em florestas.

Existem alguns trabalhos que propõem o uso de drones para coleta de pequenas quantidades de dados em uma rede de sensores [Gu et al. 2013, Abdulla et al. 2014, Jeong et al. 2015, Say et al. 2016]. A principal inovação deste trabalho é usar os drones para a coleta de grande quantidade de dados, capturados e transmitidos por dispositivos de baixo poder computacional dentro de uma floresta tropical. Por este motivo, existem dois grandes desafios para a coleta das fotos de animais silvestres. O primeiro é a transmissão de uma quantidade significativa de dados, com restrição de consumo de energia. A segunda é a transmissão de dados em um ambiente de floresta densa e fechada onde o desempenho de redes sem fio pode ficar prejudicado. Portanto, o objetivo principal deste artigo é caracterizar a transmissão de dados de dispositivos IEEE 802.11, de baixo custo, poder computacional e consumo de energia, em ambientes de florestas tropicais. Assim, o módulo 802.11 é avaliado na transmissão de dados do interior da floresta para um drone situado acima da copa das árvores. Além disso, para efeitos de comparação e melhor entendimento do efeito da floresta fechada no desempenho da comunicação, também é avaliado o desempenho do módulo em cenários internos, externos e dentro da floresta.

Apesar de alguns trabalhos já terem avaliado a transmissão do padrão IEEE 802.11 em florestas, nenhum deles avaliou dispositivos de baixo poder computacional, que tem um impacto significativo no desempenho da comunicação, como mostrado nos resultados deste trabalho. Além disso, a maioria deles realizam os testes em outros tipos de florestas, que possuem características distintas. Portanto, não foi encontrado na literatura uma

\footnotetext{
${ }^{1}$ www.lora-alliance.org

${ }^{2}$ WWW.sigfox.com
} 
avaliação da comunicação entre um dispositivo IEEE 802.11 para aplicações de IoT e um drone, através da copa das árvores de uma floresta tropical.

O restante deste artigo está organizado da seguinte forma. Na Seção 2, são apresentados os principais trabalhos relacionados à avaliação do desempenho do IEEE 802.11, bem como à coleta de dados por drones. A ideia principal do sistema de coleta de dados de animais silvestres é apresentada brevemente na Seção 3. A Seção 4 detalha os experimentos para a avaliação de desempenho do IEEE 802.11. Os principais resultados são apresentados e analisados na Seção 5. Por fim, a Seção 6 conclui o trabalho e apresenta os trabalhos futuros.

\section{Trabalhos relacionados}

Exaustivos estudos sobre redes sem fio podem ser encontrados na literatura. Seja como em [Petajajarvi et al. 2015] onde é avaliado o desempenho e alcance de uma rede LPWAN (Low-Power Wide-Area Network) em cidade, ou em [Kriara et al. 2016] que realiza testes em um ambiente de escritório de uma rede IEEE 802.11ac, avaliando a justiça na transmissão ao serem alteradas as configurações do protocolo, e ainda em [Juang et al. 2008], que avalia a capacidade do enlace de uma rede IEEE $802.11 \mathrm{~b} / \mathrm{g}$ construída em um campo de futebol americano universitário. Diversas tecnologias foram avaliadas, nas mais variadas situações e com enfoques totalmente diferentes.

Há ainda propostas de avaliações, que como nesse artigo, enfatizam o ambiente florestal. Como em [Heng Siong Lim et al. 2012] e [Mutschlechner et al. 2013] onde, no primeiro, os autores investigam o efeito da vegetação sobre uma rede IEEE 802.11n em uma floresta tropical na Malásia. Usando antenas direcionais com ganho de $19 \mathrm{dBi}, 3$ dispositivos foram dispostos na floresta à 40 m, 108 m e 174 m de distância do ponto de acesso. O objetivo desse estudo era avaliar a viabilidade de construir uma rede para levar o acesso a Internet a áreas rurais da região. No segundo trabalho, os autores avaliaram o desempenho de uma rede IEEE $802.11 \mathrm{a} / \mathrm{b} / \mathrm{g}$ entre sensores, para fins de monitoramento de morcegos. Houve a preocupação de se avaliar em diversos cenários de floresta, a saber, com pouca, nenhuma e densa vegetação. Nesse artigo, a distância de $90 \mathrm{~m}$ apresentou $100 \%$ de perdas de pacotes devido à densidade da vegetação. Foi concluído, também, que a vegetação tem um efeito muito maior no desempenho da rede do que a distância. Wang et al. [Wang et al. 2008] avaliam o desempenho de uma rede mesh que envia dados de sensores, que monitoram uma bacia hidrográfica na floresta, a um campus universitário, com uma distância total de aproximadamente $8 \mathrm{~km}$, usando as tecnologias IEEE $802.11 \mathrm{a} / \mathrm{b} / \mathrm{g}$ e IEEE 802.15.4 ao longo da rede. A rede é dividida em rede de trânsito - que leva os dados até o campus - e rede de sensores na floresta. Já Ding et al. [Ding et al. 2016] avaliam uma rede IEEE 802.15.4 em três tipos diferentes de florestas, mostrando o efeito deste ambiente na qualidade dos enlaces.

No âmbito de monitoramento usando dispositivos de Internet das Coisas, como esse artigo, o autor em [Nikhade 2015] propõe uma aplicação de monitoramento de baixo custo que usa RaspberryP $i$ como concentrador de dados dos nós sensores, entretanto usando comunicação baseada no padrão IEEE 802.15.4. Em outro sistema, proposto em [Huu-Quoc Nguyen et al. 2015], também é utilizado um RaspberryPi para monitoramento, porém ele é associado à uma câmera que transmite em tempo real as imagens do sistema de vigilância para a Internet. 
Outro trabalho interessante é a aplicação proposta em [Vasisht et al. 2017], onde é possível ver o uso de veículos não-tripulados para o auxílio na monitoração e predição de dados para cultivo e criação de gado em fazendas. Porém esta aplicação considera pequenas quantidades de dados coletadas pelos sensores. Além disso, o drone não é utilizado para coletar os dados dos sensores, mas sim para filmar a fazenda. A coleta de dados é realizada por estações base IoT, proposta no trabalho.

Não obstante os trabalhos citados acima, os autores deste artigo desconhecem o uso de drones para coleta de grande volume de dados de monitoramento em floresta utilizando dispositivos IoT - de baixo custo e baixo consumo de energia, e assim com severas limitações de processamento e capacidade de transmissão. Em consequência, nenhuma avaliação e caracterização proposta neste artigo foi encontrada.

\section{IoTWild: solução IoT para coleta de fotos de animais silvestres em florestas}

A pesquisa de animais silvestres é um tema bastante importante na área de ecologia, não somente para conhecer e entender o comportamento destes animais, mas sobretudo para preservar a sua existência. Dentro deste contexto, o monitoramento da vida silvestre nestes ambientes é fundamental para viabilizar o estudo e acompanhamento da vida destes animais. Diversos trabalhos e projetos utilizam informações para registrar a ocorrência de espécies, fazer estimativas de tamanhos populacionais ou mesmo acompanhar indivíduos específicos de espécies prioritárias, gerando conhecimento sobre hábitos, horários de atividade, áreas de vida e outros que subsidiam decisões sobre o manejo e a conservação das espécies [Steenweg et al. 2017].

Basicamente, esse tipo de monitoramento se dá por meio da instalação de cameras trap (câmeras armadilha) espalhadas pela área monitorada. Como explicado anteriormente, este sistema impõe um elevado custo para a coleta das informações capturadas. Assim, com foco em suprir esta demanda, o projeto IoTWild ${ }^{3}$ visa contribuir com a criação de uma aplicação experimental para automatizar o trabalho de coleta de dados de monitoramento de vida selvagem em floresta com o auxílio de veículos aéreos nãotripulados. O intuito é onerar o mínimo possível o sistema de monitoramento já existente, tanto em termos financeiros quanto em relação ao consumo de energia. Por isso são escolhidos equipamentos de baixo custo, no vasto universo de dispositivos de Internet das Coisas, para extraírem as imagens da câmera a as enviarem, por meio de uma rede sem fio, para outro dispositivo acoplado a um drone, como ilustrado na Figura 1.

De maneira geral, a aplicação funcionará da seguinte forma: o drone sobrevoará próximo a copa das árvores, até que algum dispositivo o identifique e se conecte a sua rede trocando as informações de suas credenciais. Uma vez que o veículo aéreo não-tripulado identifique uma nova conexão ele se estabilizará no ar, e começará a receber os dados; ele permanecerá parado até que: o dispositivo na câmera informe que não há mais dados a serem enviados ou que sua bateria sinalize baixa carga, o que ocorrer primeiro. Para que esta aplicação funcione, possíveis limitações devem ser estudadas e superadas, a saber:

- o consumo energético do dispositivo instalado para processar e enviar as imagens, de maneira que esse não eleve o número necessário de visitas de manutenção para troca de baterias;

\footnotetext{
${ }^{3}$ www.gta.ufrj.br/ velloso/projects/iot-wild/
} 


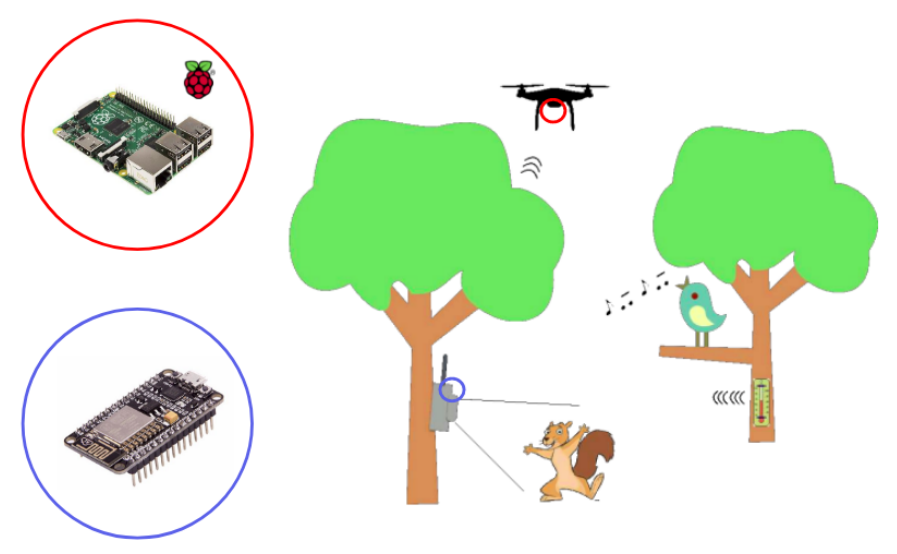

Figura 1. Cenário base da aplicação loTWild

- a autonomia da bateria do drone, que em dispositivos profissionais é de aproximadamente 15 a 20 minutos de voo, de forma que é importante transmitir o máximo possível de imagens, a fim de minimizar as recargas e os retornos do drone;

- eficiência da rede sem fio em um ambiente florestal, sendo esta construída com dispositivos de baixa consumo de energia e, por conseguinte, baixa capacidade de processamento e taxa de transmissão.

Neste artigo é avaliado esse último item, analisando assim, o desempenho de uma rede sem fio entre um dispositivo na floresta e outro acima das árvores. Conhecendo a capacidade desta rede, é possível estimar se o tempo de voo do drone será suficiente para a aplicação ser minimamente eficiente. Além disso, a caracterização da rede pode permitir a proposição de novos mecanismos e protocolos para aumentar a eficiência da transmissão.

\section{Avaliação de desempenho do módulo IoT IEEE 802.11}

O padrão IEEE 802.11 foi escolhido por oferecer uma taxa superior às outras tecnologias para IoT, e possuir dispositivos de baixo consumo de energia, como exigido pela aplicação em foco. Vale ressaltar que as fotos capturadas variam de $500 \mathrm{kB}$ à $1 \mathrm{MB}$ de tamanho. A Figura 2(a) ilustra um exemplo de uma dessas fotos. Portanto, para avaliá-lo, foram realizados 5 tipos de testes divididos em 3 cenários distintos para fins de caracterização e compreensão do desempenho dos dispositivos. O objetivo é avaliar os efeitos factuais que o ambiente, sobretudo de floresta, tem sobre a rede IEEE 802.11. Nos experimentos foram observadas as métricas padrões de rede sem fio - taxa de transmissão, alcance máximo, taxa de perda de pacotes e RSSI (Received Signal Strength Indication força do sinal recebido).

\subsection{Cenário: Floresta}

O cenário utilizado para o teste do equipamento foi a floresta do Parque Nacional da Tijuca, que constitui a maior floresta urbana do mundo ${ }^{4}$, localizada no Rio de Janeiro, devido a demanda local por um serviço que facilitasse a recuperação dos dados das câmeras que monitoram a sua fauna. O parque corresponde a uma área de 3.200 hectares que é coberta por floresta de mata atlântica. A principal diferença para outros tipos

${ }^{4}$ www. parquedatijuca.com.br 


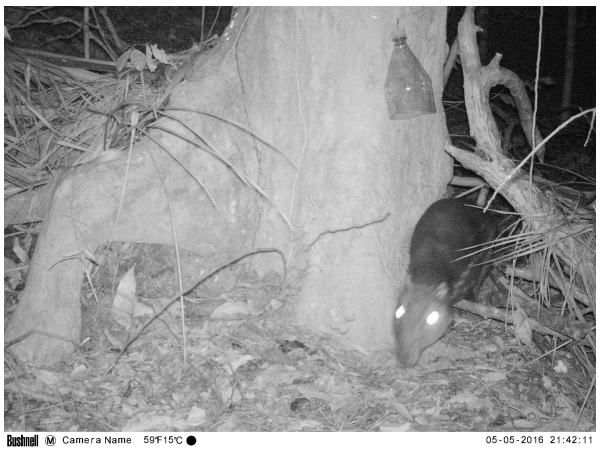

(a) Foto capturada na floresta com as câmeras de monitoramento, tamanho aproximado $600 \mathrm{kB}$

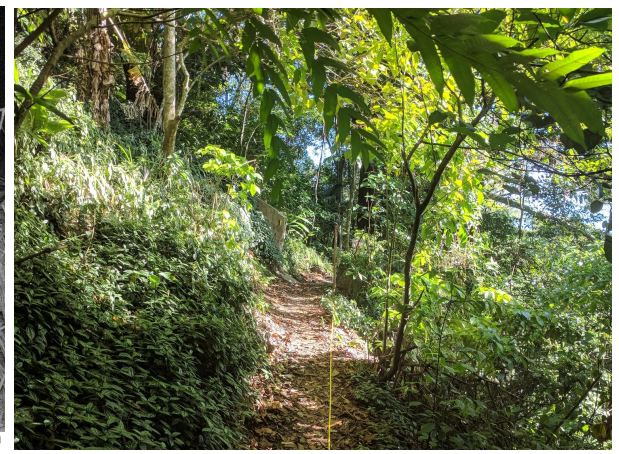

(b) Trilha onde foram realizados os testes

Figura 2. Floresta da Tijuca no Rio de Janeira caracterizada por densa vegetação e rica fauna

de florestas é que as árvores formam uma cobertura densa e contínua de vegetação que caracteriza uma mata fechada [Freitas et al. 2006], prejudicando a comunicação com o drone.

Na Figura 2(b), é possível ver parte da trilha que leva às câmeras de monitoramento. Nesse espaço foram realizados tanto os testes de transmissão com o veículo aéreo não-tripulado sobre a copa das árvores, quanto um segundo teste com ambos dispositivos da rede dentro da floresta.

\subsection{Cenários: externo e interno}

Os testes internos foram realizados no campus da Universidade Federal do Rio de Janeiro, na Ilha do Fundão. O primeiro foi realizado em um corredor de $170 \mathrm{~m}$ de comprimento cercado por salas de aula e laboratórios. O teste todo transcorreu em visada direta, ou seja, sem nenhuma obstrução na linha de transmissão dos dois dispositivos. $\mathrm{O}$ segundo formato de teste foi realizado entre duas salas de aula, de $20 \mathrm{~m}$ de comprimento cada. Cada sala mobiliada com cadeiras, mesas e outros aparatos comuns a esse tipo de ambiente. Inicialmente, os dois dispositivos foram posicionados no mesmo ambiente, em seguida um dispositivo foi movido para a segunda sala, sendo colocado no ponto mais distante em relação ao outro dispositivo. Neste segundo teste, além das barreiras comuns da sala, havia uma parede entre os dispositivos.

O cenário externo foi uma rua pouco movimentada na cidade de Petrópolis, Rio de Janeiro. Ampla e aberta, esta rua também não sofre interferência de redes sem fio da vizinhança, uma vez que é afastada de moradias. A Tabela 1 resume os cenários dos experimentos.

\subsection{Configurações/montagem do equipamento}

A intenção do projeto é ser capaz de, aproveitando os equipamentos existentes de monitoramento local, coletar imagens capturadas pelas câmeras armadilhas. Os três principais requisitos para a escolha do dispositivo de comunicação são: (i) alta taxa de transmissão; (ii) baixo consumo de energia e (iii) baixo custo. O baixo custo é importante, pois o Parque Nacional da Tijuca, por exemplo, conta com 50 câmeras armadilha 


\begin{tabular}{c|l}
\hline Experimento & \multicolumn{1}{|c}{ Descrição } \\
\hline 1 & Externo Aberto \\
2 & Interno Corredor \\
3 & Interno com Barreira \\
4 & Externo dentro da Floresta \\
5 & Dentro da Floresta com Drone \\
\hline
\end{tabular}

Tabela 1. Resumo dos Experimentos Realizados

espalhadas pela floresta; usar dispositivos mais caros nessa situação agregaria elevado custo a verba destinada ao monitoramento do parque, pois teria que ser instalado um dispositivo por câmera. Assim, foram escolhidos para os testes 3 versões do módulo WiFi ESP8266, devido ao seu custo-benefício, a saber: ESP-01, ESP-201 e ESP-12E. Os resultados apresentados nesse artigo dizem respeito aos testes realizados com esse último módulo, pois apesar de terem arquiteturas muito semelhantes ele apresentou melhores resultados em relação as outras duas versões.

O módulo de desenvolvimento ESP-12E NodeMCU, da marca DOIT, possui suporte aos padrões IEEE $802.11 \mathrm{~b} / \mathrm{n} / \mathrm{g}$ e ainda com pilhas TCP/IP e UDP integradas. Tem processador de 32 bits e clock de até $160 \mathrm{MHz}^{5}$. Diferente dos outros, esse módulo ainda possui um chip para realizar interface USB-serial e, ainda, regulador de tensão, facilitando sua programação e podendo ser alimentado por tensões entre 4,5 a $9 \mathrm{~V}$. O módulo ESP-12E nos experimentos funcionou como cliente, se conectando e enviando os dados ao ponto de acesso, este último acoplado ao drone.

Inicialmente, tanto para o ponto de acesso quanto para o cliente foram usados módulos ESP-12E, entretanto obteve-se baixíssimo desempenho, com vazão aproximada de 100 bps, e alta taxa de retransmissões. Dessa forma, optou-se por usar o microcomputador Raspberry Pi 3 como ponto de acesso, devido a sua superior capacidade de processamento e armazenamento, uma vez que o projeto visa recolher imagens de diversas câmeras. Além disso, o Rasperry não representa um gargalo em termos de consumo de energia, visto que o servidor é acoplado ao drone que possui autonomia muito inferior a do Raspberry. O microcomputador Raspberry P $i$ 3, suporta o padrão IEEE 802.11n. Tem CPU 1.2 GHz 64-bit quad-core ARMv8 ${ }^{6}$.

Cada rodada dos experimentos consiste em enviar 250 pacotes com tamanhos fixos usando os protocolos UDP e TCP do módulo ESP8266 para o Raspberry (ponto de acesso no drone). As rodadas foram repetidas diversas vezes a fim de se obter uma média para cada métrica medida. A implementação dos protocolos TCP e UDP no módulo é limitada. Observou-se que usando o protocolo TCP só era possível enviar pacotes de tamanho máximo de 2 vezes o MTU, ou seja, aproximadamente 3.000 bytes; caso fosse maior ocorria um reinício espontâneo do módulo sem fio. Semelhante limitação ocorria com o protocolo UDP, porém mais severa; pacotes maiores que o MTU (1.500 bytes) geravam a mesma falha, não havia a possibilidade de fragmentação de pacote. Outra falha que causava o reinício espontâneo no módulo era, no caso do protocolo UDP, a necessidade de um intervalo de espera para processamento de cada pacote antes do envio

\footnotetext{
${ }^{5}$ http://wiki.ai-thinker.com/esp8266

${ }^{6}$ https: / /www.raspberrypi.org/learning/hardware-guide/
} 
do próximo. Isto significa que foi necessário acrescentar este intervalo mínimo entre a transmissão de pacotes consecutivos. Diversos tempos de espera foram testados, o menor tempo necessário encontrado foi de $10 \mathrm{~ms}$ por pacote; esse valor independe do tamanho do pacote.

Em todos os testes, para evitar a influência do solo, o módulo sem fio foi posicionado a 1,30 m do chão [Mutschlechner et al. 2013]. Nos testes em terra o Raspberry P $i$ também foi posicionado a mesma distância do chão. Os tamanhos de pacote usados nos testes com o protocolo TCP foram: 128, 512, 1.024, 1.460, 2.000 e 2.920 bytes. Os mesmos tamanhos foram usados para os envios com o protocolo UDP, porém devido as limitações do módulo, não foram enviados pacotes com tamanho 2.000 e 2.920 bytes. E, ainda, como o veículo aéreo não-tripulado não faria voos em dias de chuva ou com forte vento, os testes foram realizados todos em condições parecidas, ou seja, em dias ensolarados e/ou sem fortes ventos e chuvas. No entanto, como boa parte do parque fica em uma montanha do Rio de Janeiro, não foi possível evitar totalmente rajadas de ventos momentâneas, que deslocavam o drone da sua posição de coleta, acarretando diferenças nos resultados, mesmo em testes realizados durante o mesmo dia.

Para cada teste, exceto com o drone, o procedimento adotado foi: (i) ir afastando os dispositivos até que estes, devido à distância, não conseguissem se comunicar; (ii) registrar a maior distância que houve comunicação entre eles e iniciar o envio de pacotes; (iii) aproximar os módulos, variando a distância, para verificar os efeitos na comunicação. Em todos os testes são observadas as métricas: RSSI, taxa de transmissão e as perdas na transmissão.

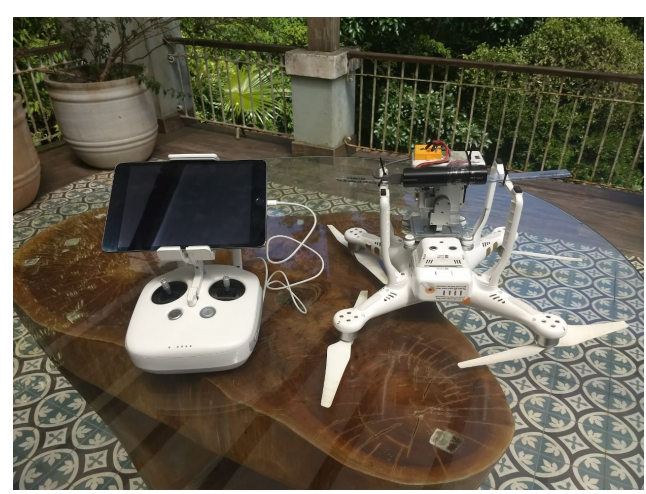

(a) Drone com Raspberry Pi acoplado

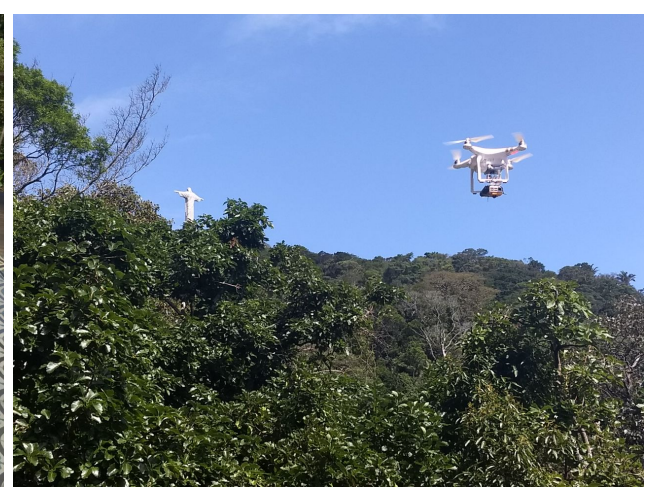

(b) Drone sobrevoando com o equipamento as árvores na Floresta da Tijuca

Figura 3. Montagem do equipamento para testes

O parque conta com um drone modelo Phantom 3 Professional com autonomia de 15 a 20 minutos completamente carregado. Nele foi acoplado o Raspberry P $i$, como mostrado na Figura 3(a), para realizar os testes de coleta sobre a copa das árvores. Diferentes dos outros experimentos, o quinto não foi até o alcance máximo de comunicação. O veículo aéreo não-tripulado sobrevoou próximo a copa das árvores, com uma distância de segurança, simulando o ambiente proposto para a aplicação IoTWild, e estabilizando ao iniciar a transmissão dos dados. 


\section{Resultados}

Nesta seção são apresentados os principais resultados referentes a cada um dos cenários avaliados. Todas as medidas representam médias com um intervalo de confiança de $95 \%$.

\subsection{Alcance máximo}

Primeiramente é avaliado o valor da relação sinal ruído a fim de determinar o alcance de cada um dos cenários, exceto o cenário com o drone.

A Figura 4 apresenta os resultados de dois dos cenários avaliados. No cenário externo é possível ver a queda do valor do $R S S I$ conforme o aumento da distância como esperado. Já no cenário interno de corredor o valor do $R S S I$ apresenta um comportamento diferente. Isto porque os corredores conduzem a energia propagada e funcionam como uma guia de onda, melhorando a propagação neste cenário.

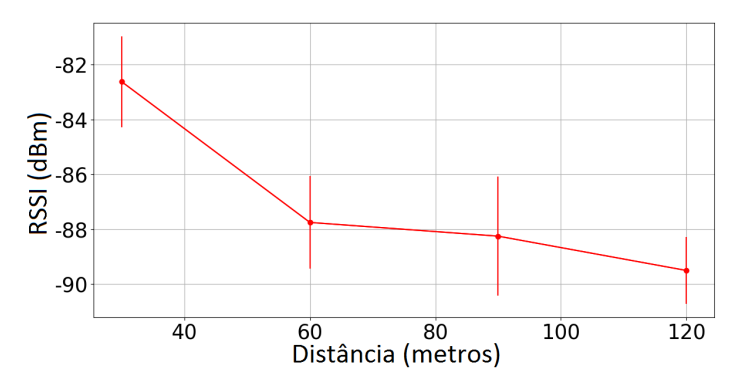

(a) Ambiente externo

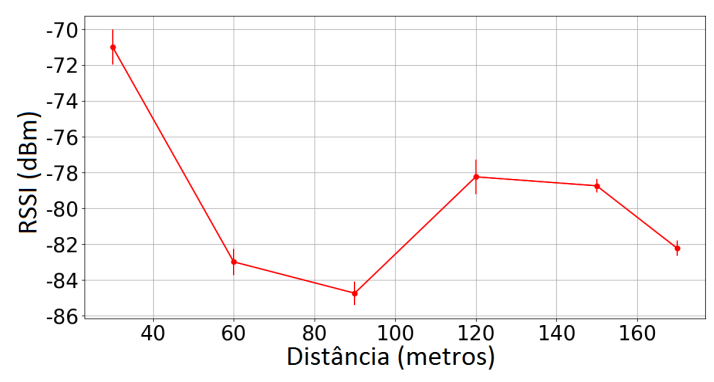

(b) Ambiente interno de corredor

Figura 4. RSSI x Alcance

A Tabela 2 mostra os alcances de cada um dos cenários avaliados. Pode-se perceber que a rede 802.11 dentro da floresta possui alcance semelhante a uma rede interna.

\begin{tabular}{c|l|c}
\hline Experimento & \multicolumn{1}{|c}{ Descrição } & Alcance (metros) \\
\hline 1 & Externo Aberto & 120 \\
2 & Interno Corredor & 170 \\
3 & Interno com Barreira & 40 \\
4 & Externo dentro da Floresta & 40 \\
\hline
\end{tabular}

Tabela 2. Alcance máximo

Na floresta com o drone, o RSSI médio foi de $-80,75 \mathrm{dBm}$ com desvio padrão de 2, 82, a uma distância de 30 metros. Como mencionado anteriormente, neste cenário não foi medido o alcance máximo, pois não fazia sentido para a aplicação em questão.

\subsection{Vazão da rede}

Nestes experimentos, buscou-se avaliar a vazão da rede utilizando os protocolos UDP e TCP. Os testes com o protocolo UDP são importantes para mostrar um limite superior da vazão, além de servir como referência para outros trabalhos que avaliam redes IEEE 802.11. Além disso, é possível caracterizar as perdas de pacotes. No entanto, a transmissão das fotos capturadas pela câmera exige confiabilidade na transmissão e 


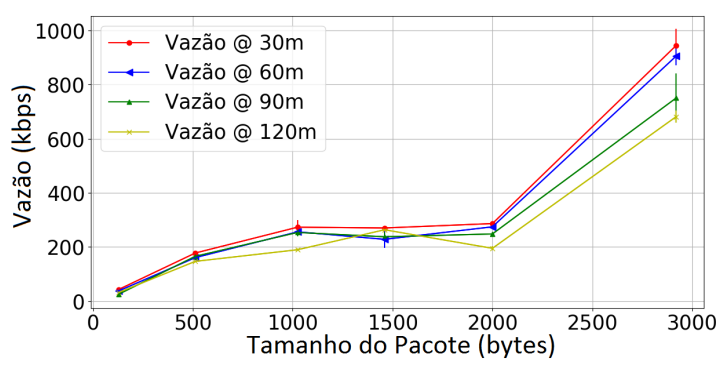

(a) TCP

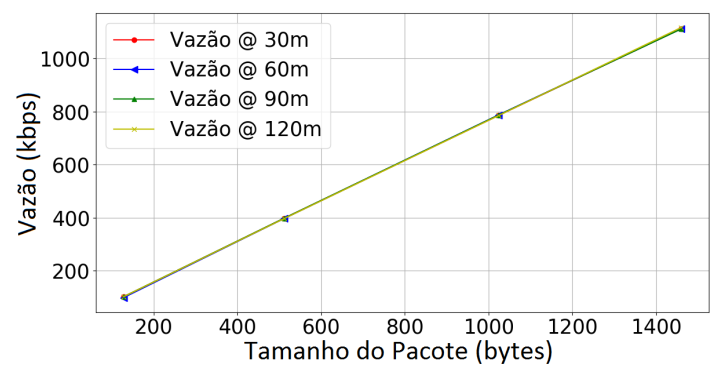

(b) UDP

Figura 5. Vazão $x$ tamanho de pacote em ambiente externo

portanto, o uso do protocolo TCP. Assim, o desempenho do protocolo TCP é fundamental para estimar a vazão que o módulo sem fio neste cenário proporcionará.

A Figura 5 ilustra o desempenho do módulo no cenário externo, onde já é possível notar uma grande diferença em relação ao desempenho de dispositivos tradicionais IEEE 802.11, isto é, sem restrições de processamento e consumo de energia. Tanto a vazão alcançada quanto o alcance atingido são inferiores aos resultados obtidos usualmente [Juang et al. 2008]. Um segundo ponto importante é verificar o efeito da restrição do intervalo de tempo mínimo entre dois pacotes, exigido pelo módulo. No UDP (Figura 5(b)) é mais nítido o efeito, pois o acréscimo de $10 \mathrm{~ms}$ a cada transmissão de pacote torna o seu tempo de transmissão praticamente desprezível, e por isso, a curva é uma reta, proporcional ao tamanho do pacote. A Figura 5(a) apresenta o resultado para o TCP. Neste caso, é interessante observar que apesar de não haver uma restrição de configurar um intervalo mínimo entre os pacotes transmitidos, o impacto da limitação do módulo de transmitir dois pacotes consecutivos permanece. Este impacto é mais visível quando colocamos o tamanho de pacote máximo permitido pelo módulo. Neste caso, ocorre fragmentação do pacote, mas claramente não há acréscimo do intervalo mínimo entre pacotes para os dois fragmentos, e portanto, a vazão atinge seu máximo. Este fenômeno ocorre em todos os outros cenários.

Por último, pode-se perceber que não houve grandes variações na vazão alcançada para as diferentes distâncias, mesmo com a queda do valor de RSSI, como mostrada na Figura 4(a). Esta queda do RSSI normalmente acarreta uma mudança de modulação do IEEE 802.11, implicando uma redução na taxa de transmissão. No entanto, esta redução não afeta a vazão do módulo, visto que o gargalo é o próprio módulo que não consegue atingir altas taxas de transmissão.

As Figuras 6 e 7 apresentam os resultados para os cenários internos. O primeiro resultado interessante é a vazão no corredor, ilustrado na Figura 6. Apesar de ser um ambiente interno, a vazão é similar ao ambiente externo, onde a transmissão sem fio é sabidamente melhor, por não estar suscetível a tantas reflexões. Como explicado na Figura 4(b), a propagação em um cenário de corredor pode ser considerada com uma onda guiada, e portanto atinge vazões iguais ou superiores a ambientes externos.

A Figura 7 mostra claramente que o resultado da vazão em um cenário interno, do tipo sala, apresenta uma vazão menor que os cenários anteriores e sobretudo que a presença de barreiras, isto é, paredes entre as salas, afeta consideravelmente a vazão do 


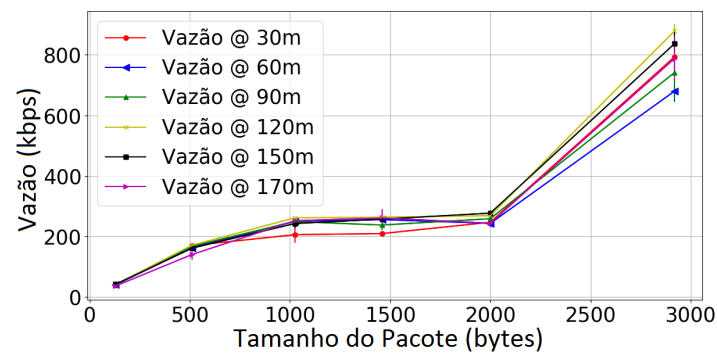

(a) $\mathrm{TCP}$

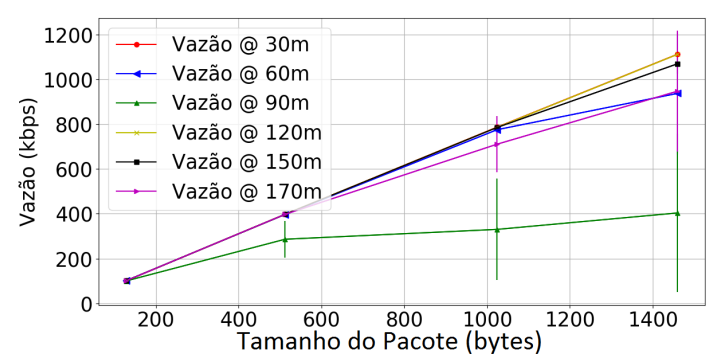

(b) UDP

Figura 6. Vazão $x$ tamanho de pacote em ambiente interno

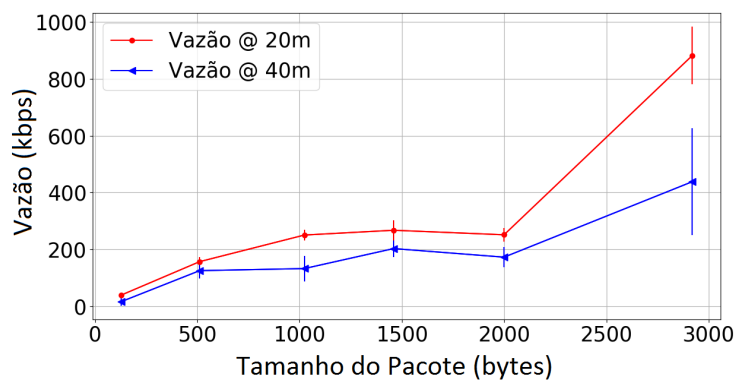

(a) TCP

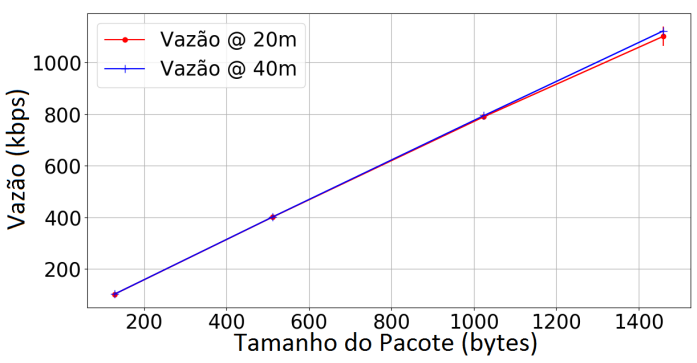

(b) UDP

Figura 7. Vazão $x$ tamanho de pacote em ambiente fechado com barreiras

TCP, que para cada perda de pacote diminui sua vazão devido às retransmissões e ao controle de congestionamento.

As Figuras 8 e 9 apresentam os resultados obtidos na floresta tropical. No primeiro experimento, onde cliente e servidor estavam dentro da floresta, a vazão e o alcance atingiram valores semelhantes ao cenário interno com barreiras. Exceto pelo desempenho do TCP que foi prejudicado pela existência de uma parede (Figura 7(a) - 40 metros). Isto significa que a transmissão em florestas densas pode ser comparado a um cenário interno.

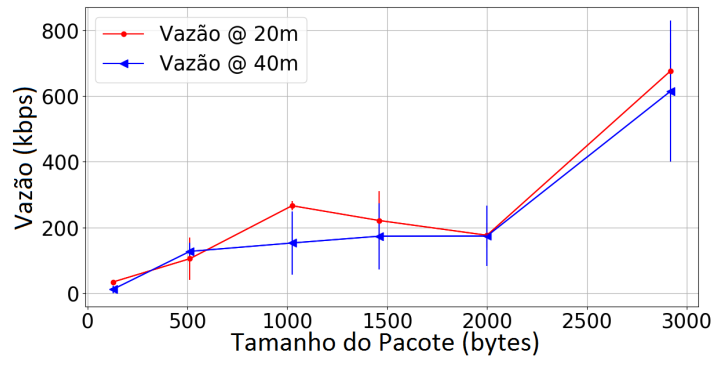

(a) TCP

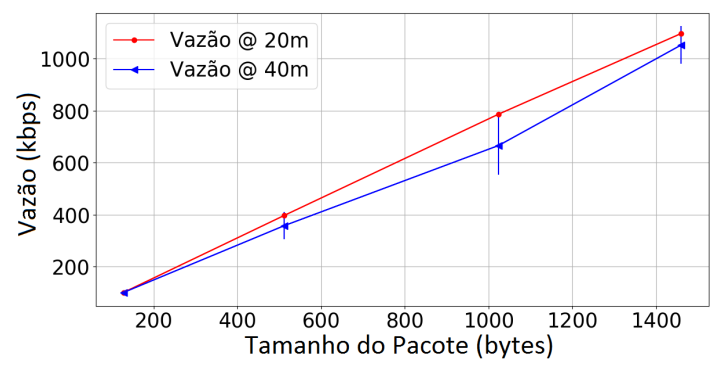

(b) UDP

Figura 8. Vazão x tamanho de pacote dentro da floresta

Finalmente, a Figura 9 mostra o desempenho do TCP e do UDP na comunicação entre o drone e o módulo 802.11 dentro da floresta. É importante notar que o desempenho do UDP foi bastante inferior aos outros cenários. Este resultado se deve principalmente pela ocorrência de um número maior de perdas de pacotes neste cenário, como será mos- 


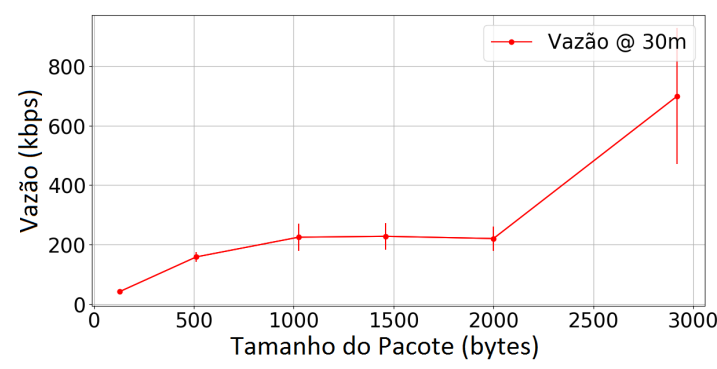

(a) TCP

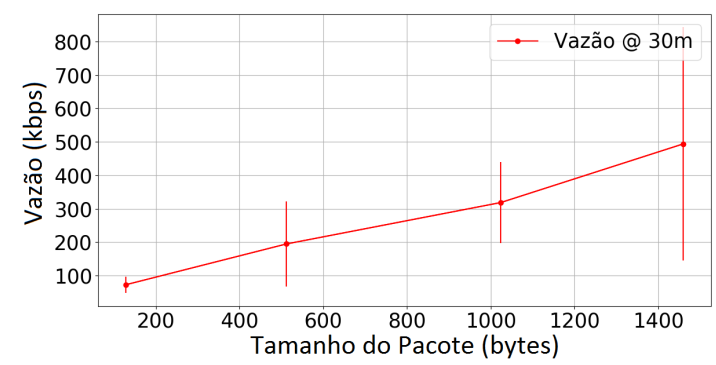

(b) UDP

Figura 9. Vazão x tamanho de pacote experimento com drone

trado em seguida. Um dos motivos para este resultado é o fato de o drone não conseguir ficar estável exatamente no mesmo lugar, devido sobretudo ao vento, e o peso extra do equipamento de comunicação acoplado, que dificultava a sua estabilidade. O resultado da vazão do TCP, ilustrado na Figura 9(a), demonstra a viabilidade da comunicação acima da copa das árvores, com um desempenho similar ao cenário interno.

\subsection{Taxa de perda de pacotes}

A partir dos experimentos da transmissão UDP com o drone, observou-se que a perda de pacotes neste cenário foi significativa. Portanto, a Tabela 3 mostra a média da porcentagem de perdas para cada tamanho de pacote. É interessante notar a alta taxa de perda de pacotes para todos os tamanhos, que aumenta conforme o tamanho do pacote, como era esperado, devido à taxa de erro de bit do meio de transmissão (BER - Bit Error Rate). Exceto para o maior tamanho de pacote, que sofreu menos perdas que os pacotes de 512 e 1.024 bytes. Neste caso específico, o vento havia diminuído consideravelmente, e por consequência, o piloto conseguiu deixá-lo mais estável. Vale ressaltar que os resultados de perdas apresentaram grande variação, com rodadas com poucas perdas e outras com mais perdas. Isto se deve principalmente a variação de posição do drone.

\begin{tabular}{c|c}
\hline Tamanho do Pacote (bytes) & Média de Perdas \\
\hline 128 & $4,8 \%$ \\
512 & $39,4 \%$ \\
1.024 & $44,8 \%$ \\
1.460 & $27,8 \%$ \\
\hline
\end{tabular}

Tabela 3. Experimento 5: Floresta com Drone

Para melhor caracterizar as perdas de pacotes na floresta, foram avaliadas as perdas de pacotes consecutivas. A Figura 10 apresenta a PMF (Probability Mass Function) e CDF (Cumulative Distribution Function) das perdas consecutivas para pacotes de 128 e 1460 bytes. Primeiramente, é interessante observar que a grande maioria dos pacotes perdidos não são consecutivos. Para pacotes pequenos, as perdas consecutivas não passa de três pacotes, enquanto que para pacotes maiores este valor pode chegar a nove. No entanto, menos de $14 \%$ das perdas de pacotes de 1.460 bytes são superiores a três pacotes consecutivos. A partir destes resultados é possível considerar a hipótese de que neste cenário o controle de congestionamento do TCP pode não ser eficiente, como já foi estudado para redes sem fio [Francis et al. 2012]. 


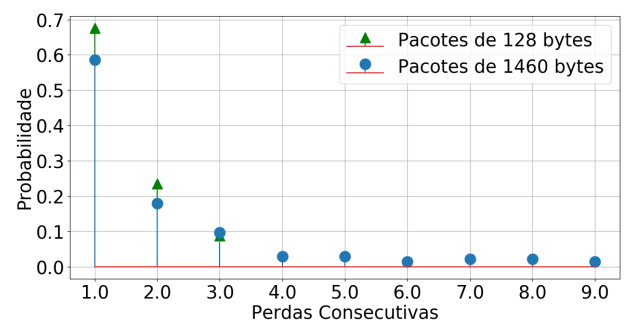

(a) PMF

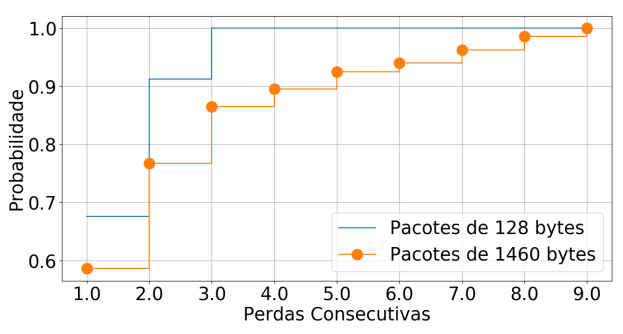

(b) $\mathrm{CDF}$

Figura 10. Perdas para o menor e maior tamanho de pacote

\section{Conclusões}

Este trabalho avalia o desempenho de uma rede IEEE 802.11 na floresta montada puramente com dispositivos IoT. Diferente de trabalhos anteriores, o enfoque é caracterizar este tipo de rede neste ambiente e verificar sua viabilidade para coleta de dados de câmeras com o auxílio de veículos aéreos não-tripulados. Para fins de caracterização, outros tipos de cenários além do florestal foram avaliados: interno e externo. Os testes mostraram que o cenário de floresta com o drone é comparável ao cenário interno com barreiras. Os resultados mostram que as limitações do dispositivos, principalmente o tempo de processamento necessário para o módulo enviar os pacotes de dados, restringem consideravelmente o desempenho da rede sem fio. Essas limitações associadas a influência da vegetação implicaram em uma diminuição na taxa de transmissão e um aumento das perdas de pacote. Entretanto, ainda que esta rede não tenha conseguido alcançar taxas ideais, ela se mostrou minimamente eficiente para coletar imagens dentro da autonomia de voo de um drone padrão, que é de 15 a 20 minutos. Portanto, estes resultados demonstram a viabilidade de automatizar a coleta de grandes volumes de dados em florestas densas.

Como trabalho futuro pretende-se avaliar o consumo de energia adicionado pelo módulo 802.11 no sistema de coleta por câmeras. Além disso, seria interessante estudar simplificações no modelo de controle de congestionamento do TCP que possam ser implementados em dispositivos de baixa capacidade de processamento a fim de melhorar o desempenho da comunicação entre o módulo e o drone.

\section{Agradecimentos}

Os autores agradecem todo o apoio recebido pelo Parque Nacional da Tijuca. Em especial, o Ernesto de Castro, diretor do parque, que disponibilizou toda a infraestrutura necessária para realizar as medidas, a Katyucha Silva, coordenadora de manejo e pesquisa e os funcionários do ICMBIO, pilotos do drone, Marcelo Mendes e Guilherme Medeiros. Este trabalho teve apoio do CNPq, CAPES, FAPERJ e processo $n^{\circ} 15 / 24494-8$, FAPESP.

\section{Referências}

Abdulla, A. E. A. A., Fadlullah, Z. M., Nishiyama, H., Kato, N., Ono, F., e Miura, R. (2014). An Optimal Data Collection Technique for Improved Utility in UAS-aided Networks. Em IEEE INFOCOM'14.

Ding, X., Sun, G., Yang, G., e Shang, X. (2016). Link Investigation of IEEE 802.15.4 Wireless Sensor Networks in Forests. Sensors, 16(7). 
Francis, B., Narasimhan, V., Nayak, A., e Stojmenovic, I. (2012). Techniques for Enhancing TCP Performance in Wireless Networks. Em ICDCSW'12.

Freitas, S. R., Neves, C. L., e Chernicharo, P. (2006). Tijuca National Park: two pioneering restorationist initiatives in Atlantic forest in southeastern Brazil. Brazilian Journal of Biology, 66(4):975-982.

Gu, Z., Hua, Q.-S., Wang, Y., e Lau, F. C. (2013). Reducing Information Gathering Latency through Mobile Aerial Sensor Network. Em IEEE INFOCOM'13.

Heng Siong Lim, Ka Kien Lo, Abbas, M., Kae Hsiang Kwong, Hashim, F., Ngoh, A., e Torshizi, S. (2012). An Investigation of Vegetation Effect on the Performance of IEEE 802.11n Technology at 5.18 GHz. Em IET ICWCA'12.

Huu-Quoc Nguyen, Ton Thi Kim Loan, Bui Dinh Mao, e Eui-Nam Huh (2015). Low cost real-time system monitoring using Raspberry Pi. Em International Conference on Ubiquitous and Future Networks.

Jeong, H., Lee, C., Ryu, J., Choi, B.-C., e Ko, J. (2015). Communicating "in the AirStudying the Impact of UAVs on Sensor Network Data Collection. Em SenSys'15.

Juang, M., Kuang-Ching Wang, e Martin, J. (2008). A Measurement Study on Link Capacity of a High Stress IEEE 802.11b/g Network. Em IEEE ICCCN'08.

Kriara, L., Molero, E. C., e Gross, T. R. (2016). Evaluating 802.11ac features in indoor WLAN. Em ACM WiNTECH'16.

Mutschlechner, M., Baldemaier, P., Handle, P., e Dressler, F. (2013). Wireless in the woods: Experimental evaluation of IEEE $802.11 \mathrm{a} / \mathrm{b} / \mathrm{g}$ in forested environments. 12. GI/ITG FACHGESPRÄCH SENSORNETZE, 60:5.

Nikhade, S. G. (2015). Wireless sensor network system using Raspberry Pi and zigbee for environmental monitoring applications. Em ICSTM'15.

Petajajarvi, J., Mikhaylov, K., Roivainen, A., Hanninen, T., e Pettissalo, M. (2015). On the coverage of LPWANs: range evaluation and channel attenuation model for LoRa technology. Em International Conference on ITS Telecommunications (ITST'15).

Say, S., Inata, H., Liu, J., , e Shimamoto, S. (2016). Priority-Based Data Gathering Framework in UAV-Assisted Wireless Sensor Networks. IEEE Sensors Journal, 16(14):5785-5794.

Steenweg, R., Hebblewhite, M., Kays, R., Ahumada, J., Fisher, J. T., Burton, C., Townsend, S. E., Carbone, C., Rowcliffe, J. M., Whittington, J., Brodie, J., Royle, J. A., Switalski, A., Clevenger, A. P., Heim, N., e Rich, L. N. (2017). Scaling-up camera traps: monitoring the planet's biodiversity with networks of remote sensors. Frontiers in Ecology and the Environment, 15(1):26-34.

Vasisht, D., Kapetanovic, Z., Won, J., Jin, X., Chandra, R., Sinha, S., Kapoor, A., Sudarshan, M., e Stratman, S. (2017). FarmBeats: An IoT Platform for Data-Driven Agriculture. Em USENIX NSDI'17.

Wang, K.-C., Venkatesh, G., Pradhananga, S., Lokala, S., Carter, S., Isenhower, J., e Vaughn, J. (2008). Building wireless mesh networks in forests. Em ACM WiNTECH'08. 